Sādhanā Vol. 38, Part 3, June 2013, pp. 397-405. (c) Indian Academy of Sciences

\title{
Low order anti-aliasing filters for sparse signals in embedded applications
}

\author{
J V SATYANARAYANA* and A G RAMAKRISHNAN \\ Department of Electrical Engineering, Indian Institute of Science, Bangalore \\ 560012 , India \\ e-mail: jvsat29@yahoo.co.in; ramkiag@ee.iisc.ernet.in
}

MS received 21 March 2012; revised 15 December 2012; accepted 29 March 2013

\begin{abstract}
Major emphasis, in compressed sensing (CS) research, has been on the acquisition of sub-Nyquist number of samples of a signal that has a sparse representation on some tight frame or an orthogonal basis, and subsequent reconstruction of the original signal using a plethora of recovery algorithms. In this paper, we present compressed sensing data acquisition from a different perspective, wherein a set of signals are reconstructed at a sampling rate which is a multiple of the sampling rate of the ADCs that are used to measure the signals. We illustrate how this can facilitate usage of anti-aliasing filters with relaxed frequency specifications and, consequently, of lower order.
\end{abstract}

Keywords. Compressed sensing; analogue-to-digital converter; streaming data; anti-aliasing filter.

\section{Introduction}

Traditionally, the restriction imposed by the Nyquist sampling theorem has been handled by the use of analog, low pass, anti-aliasing (AA) filters at the front-end of data acquisition. These analog filters, built out of passive and active analog components in most embedded designs, lead to significant utilization of space, power dissipation and add to the cost. The number of components used, is directly related to the filter order which in turn depends on the sharpness of the transition from passband to stop band. Historically, several formulas (Kaiser 1974; Oppenheim \& Schafer 1989) have been proposed and are being used to calculate the order of the filter as a function of the sampling and the cut-off frequencies. It is very clear that higher the sampling rate, the more relaxed are the restrictions on the filter. While substantial research has already been done in designing optimal filters for signals with general frequency characteristics, what remains to be explored is, if one could further optimize filter design with some additional a priori knowledge of the signal, like for example, the signal having a sparse spectral support. Sparse signals have been handled, in the past decade, by a relatively new paradigm called com-

*For correspondence 
pressed sensing (CS) (Donoho 2006; Candes \& Wakin 2008; Candes et al 2006; Marvasti et al 2009), which emphasizes on combining data acquisition and subsequent compression into a single step, thereby achieving sub-Nyquist rate sampling of the signals. While most of CS research has gone into design of undersampling architectures and efficient reconstruction algorithms, considerable focus has also been given to acquire high sampling rate reconstructions of Nyquist sampled signals, again leveraging upon the sparsity assumption. In this work, we explore the possibility of acquiring signals at higher effective sampling rate, allowing the use of low order AA filters.

\section{The filtering problem}

Consider an ensemble of signals $\underset{\sim}{s_{i}}, 0 \leq i \leq N-1$ with constituent frequencies in the band $\left[0, f_{m}\right]$. The specified sampling rate $^{1}, F$ of the analog to digital converter (ADC) for acquiring each signal must satisfy $F \geq f_{N Y Q}=2 f_{m}, f_{N Y Q}$ denoting the Nyquist rate. If the anti-aliasing (AA) filters used have a sharp transition from passband to stop band, then the analog signal is captured reasonably well. In other words, $\left(f_{\text {stop }}-f_{\text {pass }}\right) / f_{N Y Q}$, where $f_{\text {pass }}$ and $f_{\text {stop }}$ are the pass band and stop band cut-off frequencies, must be a small positive value. However, this necessitates the use of a high order filter, a requirement which is detrimental to desirable features like compactness, minimal power consumption and lower cost, typically expected in most embedded designs. Employing the same ADCs, if it were somehow possible to sample the signals at finer intervals, for example at $2 F$, then $f_{\text {stop }}$ can be greater than $f_{m}$, and lower order AA filters could be used. If the signals comprise only a sparse set of frequencies, it would be possible, under a CS architecture to reconstruct the signals using limited number of samples taken on a finer, uniform sampling grid.

\section{Compressed signal acquisition}

Before proceeding further we define the class of signals that we consider for a high sampling rate acquisition.

Definition 1: A piece-wise stationary and sparse (PSS) signal is a bandlimited signal that is a concatenation of finite, disjoint segments $\underset{\sim}{x_{k}}(t),-\infty \leq k \leq \infty$ inside each of which the Fourier transform, $\underset{\sim}{X_{k}}(j \omega)$ is sparse. More precisely, $\underset{\sim}{X_{k}}(j \omega)=0, \omega \leq-2 \pi f_{m}, \omega \geq 2 \pi f_{m}, \forall k \in \mathbb{Z}$ and the Lebesgue measure of the support of $X_{\sim}(j \omega)$ is small with respect to the full signal bandwidth, $2 \pi f_{m}$.

Definition 2: We define a reconstruction segment (RS) of order $\gamma$ as the vector $\underset{\sim}{g}(\gamma) \in \mathbb{R}^{\eta \times 1}$ obtained by uniformly sampling a PSS segment, at $\gamma$ times the specified sampling rate $F$ of

\footnotetext{
${ }^{1}$ The rate at which the ADC is specified to give its best performance.
} 
the ADC, during a finite interval $\tau_{1} \leq t \leq \tau_{2}$ lying within the PSS segment. Clearly, $\eta=$ $\left\lfloor\left(\tau_{2}-\tau_{1}\right) \gamma F\right\rfloor$. A PSS segment can be acquired and reconstructed as a series of reconstruction segments.

Let us say only $\theta<\eta$ of the $\eta$ time instants on the uniform sampling grid of order $\gamma$ are randomly chosen to be sampled. The undersampled measurement vector is given by $f=\phi \underset{\sim}{g}(\gamma)$ where $\phi \in \mathbb{R}^{\theta \times \eta}$ is the downsized identity matrix $I^{(\eta)}$ of order $\eta$ obtained by retaining only those rows in the matrix whose indices correspond to the randomly chosen sampling instants of the $\gamma$-grid. $\phi$ serves as the measurement matrix in a classical compressed sensing set-up. What then remains to be done is to get from $f$, the closest estimate $\underset{\sim}{g}$ of $g$, given that it has sparse spectral support. We omit the superscript $\gamma$, for simplicity of notation. Compressed sensing literature offers a host of recovery algorithms (Chen et al 1999; Tropp \& Gilbert 2007; Donoho et al 2012; Cormode \& Muthukrishnan 2006) for solving this problem, the most popular being those based on Basis Pursuit (Chen et al 1999) and orthogonal matching pursuit (Tropp \& Gilbert 2007). In our previous work (Satyanarayana \& Ramakrishnan 2011), we have highlighted the inadequacy of some of the existing recovery methods for reconstructing general signals with frequencies that are non-integral multiples of the fundamental DFT frequency. In this work we have discussed the merits and demerits of these solutions and provided empirical evidence of better performance by a method based on eigen decomposition proposed in Duarte \& Baraniuk (2012). This method employs the root-MUSIC algorithm for finding out the component frequencies in the signal. MUSIC obtains the eigen values and the eigen vectors of the signal autocorrelation matrix and evaluates a score function that returns the specified number of largest score function peaks as the frequencies present in the signal. The expected number $K$ of component frequencies in the signal is fed as input to the algorithm. Root-MUSIC is an extension of MUSIC which calculates the peaks from the zeros of a polynomial that depends on the noise subspace eigen vectors.

Like in greedy CS methods, the $K$ frequencies are obtained iteratively. The initial residue $r_{0}$ is equal to the measurement vector $f$. In the subsequent iterations, the residue is given as

$$
r_{j}=f-\phi \hat{g}_{j-1}
$$

where $r_{j}$ is the residue in iteration $j, \phi$ is the measurement matrix, $f$ the measurement vector and $\hat{g}_{j-1}$ the estimate of the reconstruction segment in the previous iteration. An intermediate estimate is calculated from the residue.

$$
\underset{\sim}{h}=\underbrace{\hat{g}_{j-1}}_{\sim}+\phi^{T} r_{\sim} .
$$

The intermediate estimate is fed to the Root-MUSIC algorithm as input along with the expected number $K$ of component frequencies. The prominent frequencies $\hat{\omega}_{k}$ and the corresponding coefficients $\hat{a}_{k}, 1 \leq k \leq K$ are returned by the algorithm from which the final estimate for the $j^{\text {th }}$ iteration is calculated as the linear combination of the $K$ chosen sinusoids.

$$
\underline{\underline{g}}_{\sim}=\sum_{k=1}^{K} \hat{a}_{k} \mathbf{e}\left(\hat{\omega}_{k}\right) .
$$




\section{High sampling rate reconstruction scheme}

\subsection{Streaming data acquisition}

Most CS recovery algorithms focus on finite length signals which are reconstructed off-line with sub-Nyquist number of samples. In our approach, continuous streaming data is acquired, in real time, as finite length blocks defined previously as reconstruction segments. The RSs are compressively sampled and reconstructed using the MUSIC based algorithm described in the last section. Interesting methods have been proposed by Boufounos \& Asif (2010) and Mishali \& Eldar (2009) for dealing with infinite-dimensional signals to avoid blocking artifacts introduced due to finite length blocks. The method we propose can incorporate these reconstruction algorithms. However, we choose to restrict to the finite dimensional reconstruction algorithm, since our interest is mainly in achieving higher sampling rate to facilitate use of low order AA filters.

Our approach is based on the multiplexed signal acquisition architecture, operating on streaming data, proposed in our previous work (Satyanarayana \& Ramakrishnan 2010). Each of the signals is acquired as a series of overlapping reconstruction segments. Thus, each new RS that is sampled has a significant overlap with the previous RS. In the region of overlap, all the previously reconstructed samples of the grid, of chosen order $\gamma$ (from the previous RS), are taken as it is. The non-overlapping portion is compressively sampled, that is, sub-Nyquist number of samples are taken at random instants of time as explained in section 3. After each $\mathrm{RS}$ is reconstructed, the deviation between the reconstructed signal values and the actual measured values at the few random instants, in the non-overlapping portion, where samples have been taken, is calculated. If the deviation exceeds a small threshold, then the RS would have crossed the boundary of a new PSS segment. The first RS after the detection of a PSS boundary is reconstructed without overlap. Subsequently, the reconstruction segments shall again overlap. The process continues until the next boundary is detected. Small reconstruction error at PSS boundaries, is unavoidable. This is usually small due to significant overlap between consecutive RSs.

By virtue of the overlap between RSs, it is not necessary to have exact a priori knowledge of the PSS segment boundaries since the reconstruction error is restricted to the small nonoverlapping portion of the RS falling on the PSS boundary. This is because each PSS segment is reconstructed as several overlapping RSs and most of the samples in an RS are obtained from the overlapping portion (say 80 percent) which would have been already reconstructed as part of the preceding RS. The new samples are obtained by direct measurement on the signal in the nonoverlapping portion (the remaining 20 percent). The absence of beforehand information of the PSS boundaries does not cause significant error since any PSS segment boundary will always fall in the non-overlapping portion of the last RS within a PSS segment. Since the non-overlapping portion is small, the contribution of the samples from this portion in the reconstruction of the last RS is small and therefore, the reconstruction error due to the non-stationarity is small and mostly confined to the non-overlapping portion of the RS. The reconstruction error though small, would have crossed the threshold for real-time detection of PSS boundary. Once the boundary is detected, the very first RS in the new PSS would have no overlap with the previous RS. Overlap will resume from the second RS onwards. However, a rough estimate of the minimum length of any PSS segment during the data acquisition is required. In other words, there needs to exist an assurance that the length of any PSS segment will certainly not be less than this value. This is to ensure that the calculated length of the RS (Satyanarayana \& Ramakrishnan 2010) is not more than the length of any PSS segment as this will cause the very first RS (in a new PSS) itself to fall on a PSS boundary, thereby causing significant reconstruction error. 


\subsection{The architecture}

Given the specified sampling rate of each of the $N$ ADCs as $F \mathrm{~Hz}$, let each ADC operate on clocks which have the same period $T=1 / F$, but are phase shifted from each other by $\tau$. In other words, if the acquisition starts at time $t$, the $i^{t h}$ ADC, $0 \leq i \leq N-1$, operates at the time instants: $t+i \tau, t+i \tau+T, t+i \tau+2 T \ldots$ and so on. If we choose $\tau=T / N$, we have a data acquisition system, employing $N$ ADCs, operating on a uniform sampling grid with a sampling interval of $T / N$ or equivalently an effective sampling frequency of

$$
F_{e f f}=N F \text {. }
$$

The finer uniform sampling grid, of order $\gamma=N$, is available to all the $N$ signals. During acquisition, time instants are randomly chosen from the finer grid provided by all the $N$ ADCs together to collectively sample the $N$ analog signals. This requires each ADC to be able to multiplex between the different analog signals in real time, which is practically realizable due to the presence of built-in multiplexers in commercially available ADCs. Figure 1 shows the data acquisition scheme that we propose in this work. The shaded section in the figure, which is the analog section, consists of $N$ ADCs together with the corresponding $N$ multiplexers. $N$ analog signals are input to the system. Each of the signals, after passing through AA filter, is routed to every analog multiplexer.

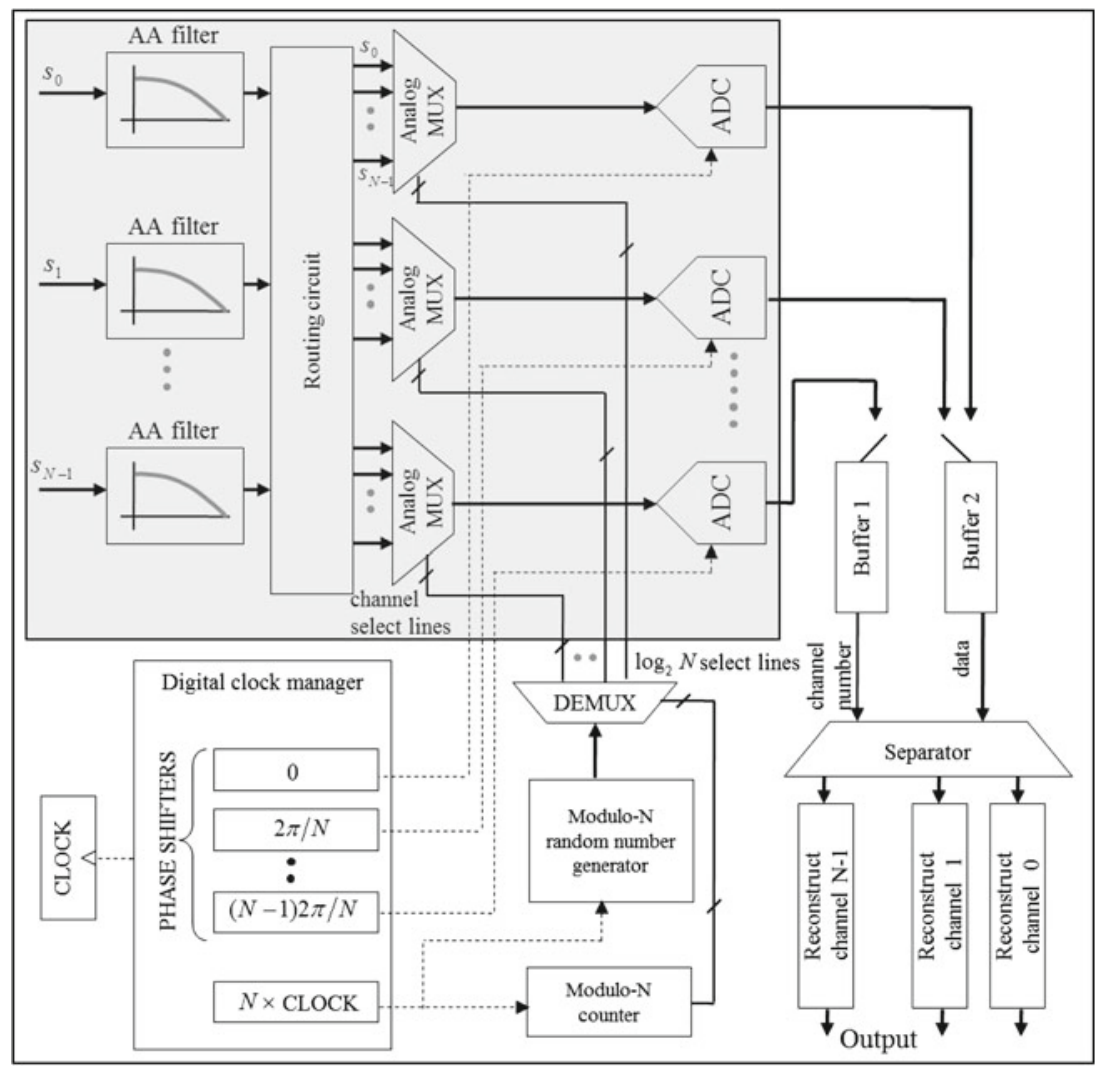

Figure 1. Compressed sensing architecture for acquiring signals with relaxed specifications for AA filter. 
The rest of the design (the unshaded region) is digital and can be implemented in a small size, low cost FPGA. The Digital Clock Manager (DCM) is a standard block commonly implemented in commercially available FPGAs. The DCM generates $N$ phase shifted versions of the input clock of $F \mathrm{~Hz}$, in the range 0 to $(N-1) 2 \pi / N$, which are input to the ADCs. The DCM also generates another clock whose frequency is $N F$, which is input to a modulo-N counter and a modulo-N random number generator. The modulo- $\mathrm{N}$ random number generator outputs a random number between 0 and $N-1$ at every tick of its clock input for choosing the analog channel to be sampled. By using a proper seed, care is taken that over sufficiently long interval of acquisition, each analog channel gets an equal share of the time instants when it is sampled. The modulo-N counter releases counts from 0 to $N-1$ in succession, such that the demultiplexer routes the number of the analog channel to be sampled to the analog multiplexers of successive ADCs, in synchronization with their respective clocks. The analog multiplexer of the ADC, which gets a clock tick, routes the chosen analog signal to the ADC.

Thus while each of the individual ADCs operate at their specified sampling rate of $F \mathrm{~Hz}$, the collective acquisition takes place at $N F \mathrm{~Hz}$. The process of acquisition and reconstruction of the signals takes place in a series of acquisition cycles. There are two digital buffers which store the samples collected by all the ADCs together. During any acquisition cycle, one of the buffers is active, into which the ADCs deposit the samples collected by them in succession. The other buffer, which contains the samples collected in the previous acquisition cycle, is read by the separator. The separator separates the samples into the individual channels making use of the random sequence generated by the modulo-N random generator, that is fed to it at the end of an acquisition cycle. For any channel, as soon as time corresponding to an RS has elapsed, the collected samples are fed to the CS reconstruction block, the output of which is the reconstructed signal. The length of the acquisition cycle, which decides the size of the buffers, must cater for the estimated worst case execution time of reconstruction in the cycle where all the signals have a complete RS available for reconstruction.

\subsection{A note on the complexity of the method}

Since the set-up operates on streaming data, the execution of the algorithm is independent of the number of PSS segments. The main chunk of computation lies in the compressed sensing reconstruction based on the Root-MUSIC algorithm. This computation time reduces if the size of each RS is small, or in other words there are more number of RSs within each PSS segment. However, too small an RS would cause the sparsity assumption, required for CS reconstruction, to fail. A trade-off is sought as explained in our previous work (Satyanarayana \& Ramakrishnan 2010). The computation load increases only linearly with the number of signals, $N$ since the reconstruction of each signal is independent of the other. On the other hand, as $N$ increases, the effective sampling rate $F_{\text {eff }}$ of each signal increases (4) due to availability of an $N$ times finer sampling grid. The increase in $F_{\text {eff }}$ far above the Nyquist rate of each signal, will only be of marginal benefit since after a point the reduction in the order of the AA filter, due to relaxed frequency specifications will not be significant.

\section{Simulation and results}

We have considered, for simulation, the simple case of acquiring two signals with the frequency characteristics shown in table 1. Each signal is a concatenation of PSS segments with durations greater than $10 \mathrm{~ms}$. Within each PSS segment, there are three frequency components. For both 
Table 1. Frequency characteristics of test signals.

\begin{tabular}{lllll}
\hline \multicolumn{2}{c}{ Signal 1 } & & \multicolumn{2}{c}{ Signal 2 } \\
\cline { 1 - 2 } \cline { 5 - 5 } Time $(\mathrm{ms})$ & Frequencies $(\mathrm{KHz})$ & & Time $(\mathrm{ms})$ & Frequencies $(\mathrm{KHz})$ \\
\hline $0-12.5$ & $3.68,8.14,13.46$ & & $0-14.1$ & $1.23,3.4,7.9$ \\
$12.5-29.3$ & $2.82,3.95,11.4$ & & $14.1-28.2$ & $2.56,3.8,13.5$ \\
$29.3-42.8$ & $1.5,4.11,5.8$ & & $28.2-46.5$ & $4.45,6.53,10.9$ \\
$42.8-57.4$ & 2.548 .5914 .5 & & $46.5-59.5$ & $3.13,6.66,12.54$ \\
$\geq 57.4$ & $1.54,2.71,7.8$ & & $\geq 59.5$ & 3.628 .3412 .81 \\
\hline
\end{tabular}

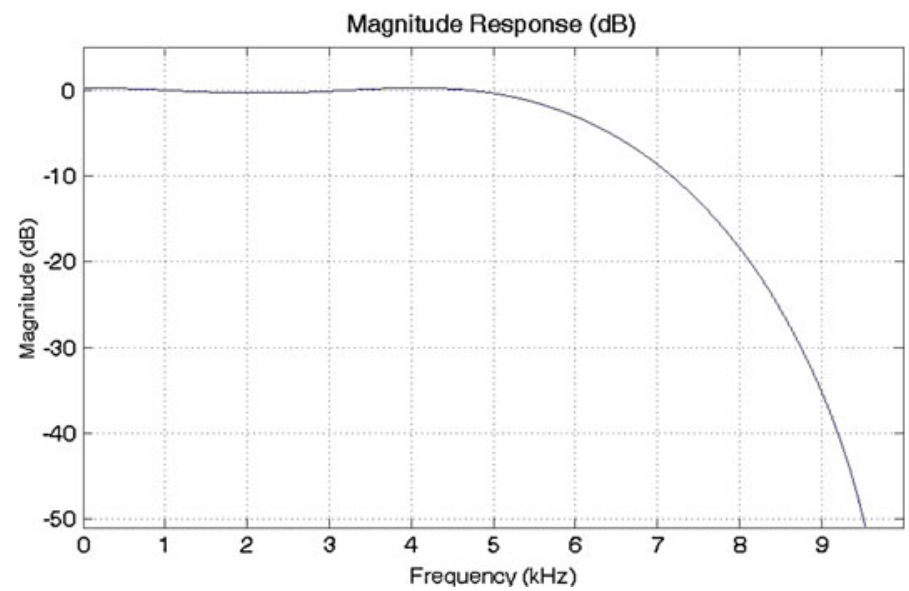

Figure 2. Magnitude response of FIR filter of order 8 .
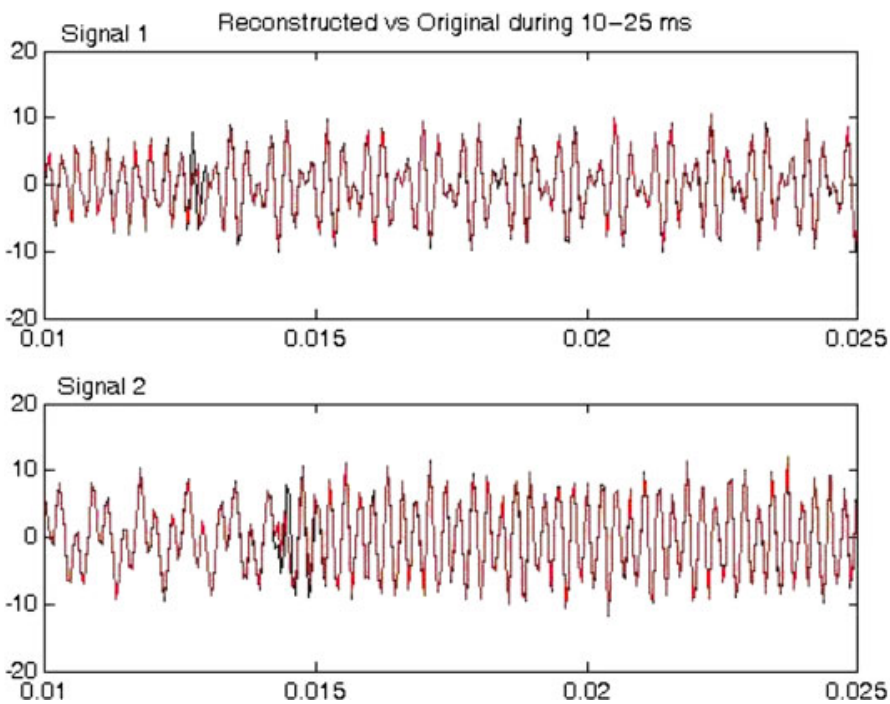

Figure 3. Reconstructed (red) vs original (black) for two signals. 
signals, the region of interest to the application is $0-5 \mathrm{kHz}$, the content above which can be filtered out. In a classical data acquisition set-up, we need to employ an AA filter with a cut-off at around $5 \mathrm{KHz}$ and sample at a rate above the Nyquist rate of $10 \mathrm{KHz}$. For a sampling rate of $12 \mathrm{KHz}$ and $f_{\text {pass }}$ and $f_{\text {stop }}$ equal to $4.5 \mathrm{KHz}$ and $5.5 \mathrm{KHz}$, respectively it is required to use an equiripple finite impulse response (FIR) filter of order 30. As $N=2$, with the data acquisition scheme proposed in this work, using two ADCs with specified sampling rates of $F=10 \mathrm{KHz}$, we get an effective sampling rate of $F_{\text {eff }}=20 \mathrm{KHz}$. This in turn implies that we can afford to choose $f_{\text {pass }}=4.99 \mathrm{KHz}$ and $f_{\text {stop }}=9.9 \mathrm{KHz}$, while preserving the signal content below $5 \mathrm{KHz}$, without any aliasing effect. The order of the AA filter with the relaxed frequency specifications is only 8 . The magnitude response of such a filter is shown in figure 2 . The reconstructed signal is plotted against the original signal in figure 3 . The close match between the reconstructed and the original for both the signals is an empirical evidence of performance. The deviation in the reconstruction for signal 1 at around $12.5 \mathrm{~ms}$ and the same for signal 2 at around $14.1 \mathrm{~ms}$ can be justified by the existence of PSS boundaries.

\section{Conclusion}

In this work, we have proposed an architecture for capturing sparse signals, in a way that reduces the order of the AA filter at the front end. Since the AA filter is part of the analog circuitry, this enhancement can have a significant reduction in the number of passive components used for realizing the filter, thereby scoring on compactness, power dissipation, cost, reliability and maintainability. Although the scheme is based on the sparsity assumption, it has enormous potential to be applied in a general situation too, provided the number of frequency components that are actually of interest to the application are limited. The paper reports simulation results for a two signal ensemble. However, in a more general setting, one can have multiple signals, for example more than five signals, in which case, the focus is more on obtaining high sampling rate reconstructions than a reduction in the filter order. The blocks in the design have been chosen such that most of them can be realized in a low cost FPGA that is invariably already included in most embedded designs for handling glue logic. The same is true for the multiplexers which are part of most commercially available ADCs.

\section{References}

Boufounos P and Asif M S 2010 Compressive sampling for streaming signals with sparse frequency content. Proc. of $44^{\text {th }}$ Annual Conf. Information Sciences and Systems (CISS), 1-6

Candes E, Romberg J and Tao T 2006 Robust uncertainty principles: exact signal reconstruction from highly incomplete frequency information. IEEE Trans. Inform. Theory 52(2): 489-509

Candes E and Wakin M 2008 An introduction to compressive sampling. IEEE Signal Proc. Magazine 25(2): $21-30$

Chen S S, Donoho D L and Saunders M A 1999 Atomic decomposition by basis pursuit. SIAM J. Sci. Comput. 20(1): 33-61

Cormode G and Muthukrishnan S 2006 Combinatorial algorithms for compressed sensing. Proc. of the $40^{\text {th }}$ Annual Conference on Information Sciences and Systems, 198-201

Donoho D L 2006 Compressed sensing. IEEE Trans. Inform. Theory 52(4): 1289-1306

Donoho D L, Tsaig Y, Drori I and Starck J 2012 Sparse solution of undetermined linear equations by stagewise orthogonal matching pursuit. IEEE Trans. Inform. Theory 58(2): 1094-1121

Duarte M F and Baraniuk R G 2013 Spectral compressive sensing. Applied and Computational Harmonic Analysis 35(1): 111-129 
Kaiser J F 1974 Nonrecursive digital filter design using the - sinh window function. Proc. IEEE Symp. Circuits and Systems, 20-23

Marvasti F, Amini A, Haddadi F, Soltanolkotabi M, Khalaj B H, Aldroubi A, Holm S, Sanei S and Chambers J 2012 A unified approach to sparse signal processing. EURASIP Journal on Advances in Signal Processing. doi:10.1186/1687-6180-2012-44

Mishali M and Eldar Y 2009 Blind multiband signal reconstruction: Compressed sensing for analog signals. IEEE Trans. Sig. Proc. 57: 993-1009

Oppenheim A V and Schafer R W 1989 Discrete-time signal processing. Prentice-Hall, 458-562

Satyanarayana J V and Ramakrishnan A G 2010 MOSAICS: Multiplexed optimal signal acquisition involving compressed sensing. Proc. International Conference on Signal Processing and Communications (SPCOM), $1-5$

Satyanarayana J V and Ramakrishnan A G 2011 Multiplexed compressed sensing of general frequency sparse signals. Proc. of International Conference on Communications and Signal Processing, 423-427

Tropp J and Gilbert A 2007 Signal recovery from random measurements via orthogonal matching pursuit. IEEE Trans. on Information Theory 53(12): 4655-4666 